\title{
Las tres perspectivas de la Reserva Nacional Allpahuayo Mishana, Loreto 2019
}

\author{
The three perspectives of the Reserva Nacional Allpahuayo Mishana, \\ Loreto 2019
}

\section{As três perspectivas da Reserva Nacional Allpahuayo Mishana, Loreto 2019}

\author{
Marisol Aguirre Nieri $^{1}$ \\ Universidad Nacional Mayor de San Marcos \\ marisol.aguirre@unmsm.edu.pe
}

\begin{abstract}
RESUMEN
Este trabajo etnográfico tiene como objetivo analizar las tres distintas perspectivas y sus relaciones de los actores de la Reserva Nacional Allpahuayo Mishana (RNAM). Por lo cual, para la obtención de esta información, se llevó a cabo las entrevistas y observaciones en un voluntariado en la RNAM, donde mi persona fue guardaparque voluntaria, llegando así a recolectar una información de investigación exploratoria. Para lograr el objetico se analizó las entrevistas y observaciones recolectadas en campo; posteriormente se procedió a desarrollar la metodología del mapeo de actores según Esteban Tapella. Analizada esta información, se concluye que las perspectivas de los tres actores: los pobladores de las comunidades campesinas, los guardaparques oficiales y la jefatura de la RNAM; manifiestan posiciones divergentes, provocando algunos conflictos para un desarrollo en conjunto de la RNAM.
\end{abstract}

\begin{abstract}
This ethnographic work aims to analyze the three different perspectives and their relationships of the actors of the Reserva Nacional Allpahuayo Mishana (RNAM). Therefore, to obtain this information, interviews and observations were carried out in a volunteer at the RNAM, where my person was a volunteer park ranger, thus collecting information from exploratory research. To achieve the objective, the interviews and observations collected in the field were analyzed; later, the actor mapping methodology according to Esteban Tapella was developed. After analyzing this information, it is concluded that the three actors, despite maintaining a relationship with each other in the same natural area, have different perspectives, causing some conflicts for a joint development of the RNAM.
\end{abstract}

\section{RESUMO}

Este trabalho etnográfico tem como objetivo analisar as três diferentes perspectivas e suas relações dos atores da Reserva Nacional Allpahuayo Mishana (RNAM). Portanto, para a obtenção dessas informações, foram realizadas entrevistas e observações em um voluntário da RNAM, onde fui guarda florestal voluntário, coletando informações de pesquisa exploratória. Para atingir o objetivo, foram analisadas as entrevistas e observações coletadas em campo; posteriormente, foi desenvolvida a metodologia de mapeamento de atores segundo Esteban Tapella. Após a análise dessas informações, conclui-se que os três atores, apesar de manterem uma relação entre si na mesma área natural, possuem perspectivas diferentes, gerando alguns conflitos para o desenvolvimento conjunto da RNAM.

1 Estudiante de la Escuela Profesional de Geografía.

Recibido: 12/04/2020 - Aceptado: 03/05/2020 - Publicado: 28/08/2020

Citar como:

Aguirre, M. (2020). Las tres perspectivas de la Reserva Nacional Allpahuayo Mishana, Loreto 2019. Espiral, revista de geografías y ciencias sociales, 2(3), 133 - 148. http://dx.doi.org/10.15381/espiral.v2i3.18453 
PALABRAS CLAVE: etnografía; actores; RNAM.

KEYWORDS: ethnography; actors; RNAM.

PALAVRAS-CHAVE: etnografia; atores; RNAM.

\section{Introducción}

Lo que primero podemos pensar sobre la "perspectiva" o "punto de vista" de algún tema, un acontecimiento o un hecho es por la previa información que has obtenido o por la vivencia que has presenciado directa o indirectamente. Es así que varias personas pueden tener diferentes puntos de vista de un mismo escenario. Estos puntos de vista pueden ser convergentes o divergentes. Por lo cual el objetivo principal del presente trabajo es la descripción de las 3 distintas perspectivas y sus relaciones de los actores de la Reserva Nacional Allpahuayo Mishana.

La perspectiva es definida por la Real Academia española (RAE), en la acepción 6, como: "visión, considerada en principio más ajustada a la realidad, que viene favorecida por la observación ya distante, espacial o temporalmente, de cualquier hecho o fenómeno"2. En otras palabras, en los hechos existe un actor que interpreta esta realidad, donde en esta se manifiesta en un espacio y en un tiempo. La interpretación de la realidad se puede desarrollar estando directamente involucrados. Como sostiene el autor, "the angle of considering things which shows us the opinion or feelings of the individuals involved in a situation"3 (Anónimo, 2018: 2). Para un mejor entendimiento, nos dice Hautamaki (2015) que "Time intervals themselves are taken to represent points of view" representaciones de los puntos de vista en el tiempo son cambiantes. Con ello es necesario mencionar que el intervalo de tiempo de recolección de datos del presente trabajo es durante los meses de enero, febrero y marzo del año 2019.

Para describir o interpretar los puntos de vista, hay que entender que se desarrolla a través de los actores sociales, según Tapella (2007:) nos indica que "pueden ser personas, grupos u organizaciones que tienen interés en un proyecto o programa". Por lo que se ha determinado tres actores sociales en el ámbito de estudio: las personas de las comunidades campesinas, los guardaparques oficiales y la jefatura de la Reserva Nacional Allpahuayo Mishana que tienen el interés de aportar al presente trabajo etnográfico.

Este espacio ocupado donde se interrelacionan los actores es la Reserva Nacional Allpahuayo Mishana (RNAM). Se encuentra políticamente en la región de Loreto, en la provincia de Maynas, donde intersecta tres distritos: Iquitos, Alto Nanay y San Juan Bautista (ver figura 1).

\section{Los Puestos de control y vigilancia y las comunidades campesinas de la RNAM}

Existen tres puestos de control y vigilancia (PCV) en toda la RNAM: Yarana, Irapay y Varillal, de las cuales solo he permanecido en los dos primeros puestos mencionados.

Las comunidades campesinas han sido creadas antes del año 1982, es así que, según el Diagnóstico del Proceso de Actualización del Plan Maestro (2013-2018), indica que existen 6 comunidades, por lo que faltó registrar a la comunidad de Nueva Esperanza

2 Real Academia Española (2001). Diccionario de la lengua española (2001). Disponible en: http://lema.rae.es/drae2001/ srv/search?id=PBcqiKUvlDXX2s6mofda [Fecha de consulta: 14/03/2019].

3 Anónimo (2018). Definition of point of view (2019). Traducción al español: "ángulo de considerar cosas que nos muestra la opinión o los sentimientos de las personas involucradas en una situación”. Disponible en: https://www.charlesclarke. org.uk/pdf-book/point-of-view-literature-definition[Fecha de consulta: 14/03/2019].

4 Hautamaki, A. (2015). Change, Event, and Temporal Points of view. ResearchGate. Traducción al español: "los intervalos de tiempo se toman para representar puntos de vista". 
al preguntar al guardaparque Germán contrastando después en el Instituto Nacional de Estadística e Informática (ver tabla 1). Por otro lado, la RNAM es creada posteriormente, precisamente el 4 de marzo de $199^{5}$ (ver figura 2).

En síntesis, existen 7 comunidades campesinas y 3 puestos de control de vigilancia en la RNAM (ver figura 2).
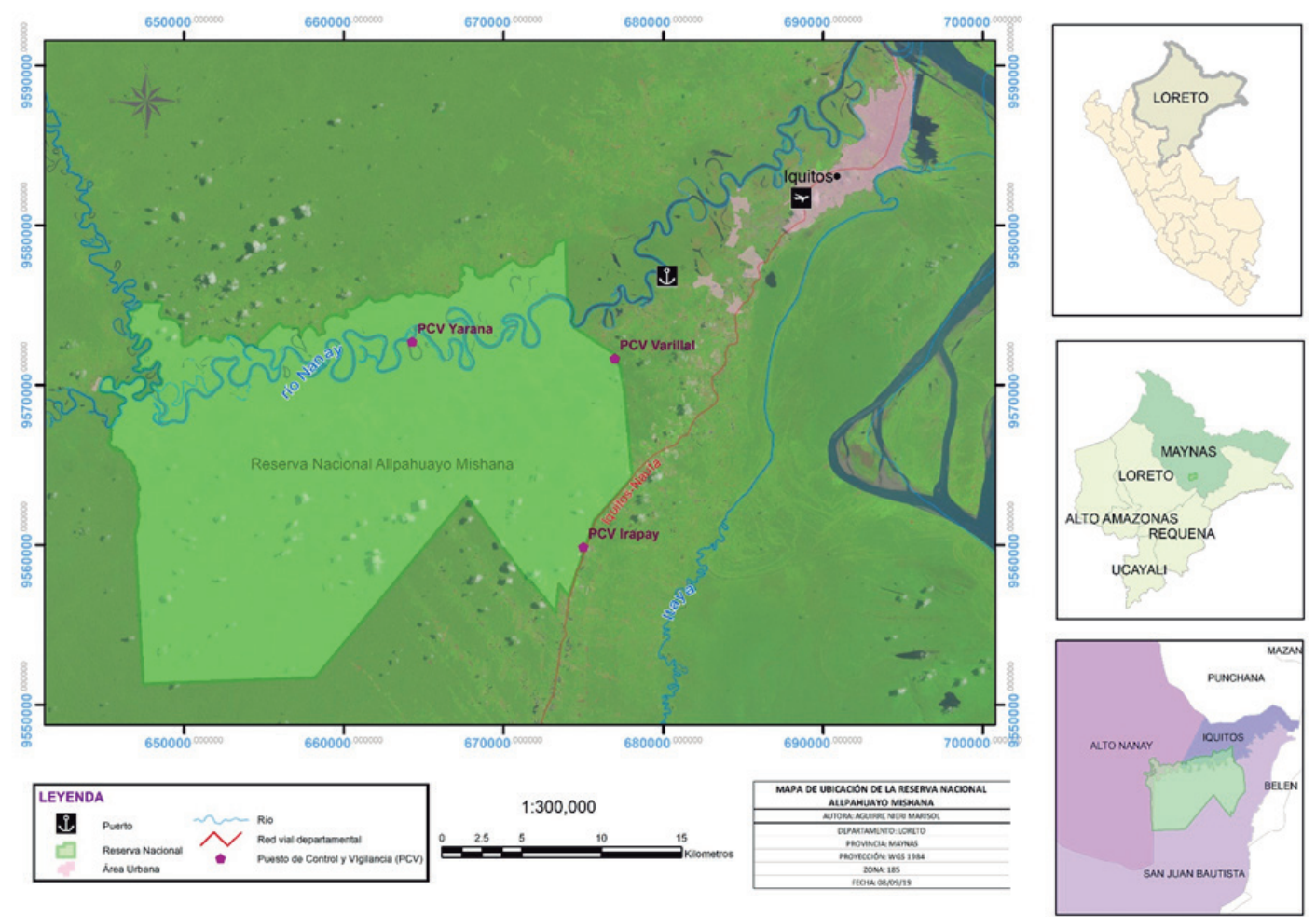

Figura 1 - Mapa de ubicación de la RNAM.

Fuente: Elaborado por Aguirre (2019)

Tabla 1. Tabla de población por año y antigüedad de las comunidades

\begin{tabular}{ccccc}
\hline Centros poblados & $\begin{array}{c}\text { Población (hab.) } \\
\mathbf{2 0 0 2}\end{array}$ & $\begin{array}{c}\text { Población (hab.) } \\
\mathbf{2 0 1 0}\end{array}$ & $\begin{array}{c}\text { Población (hab.) } \\
\mathbf{2 0 1 7}\end{array}$ & Antigüedad (años) \\
\hline San Martín & 31 & 88 & 25 & 39 \\
Mishana & 57 & 55 & 34 & 65 \\
Yuto & 20 & 48 & 23 & 28 \\
El Porvenir & 47 & 68 & 42 & 55 \\
15 de abril & 60 & 84 & 20 & 68 \\
Anguilla & 30 & 54 & 22 & 38 \\
Nueva Esperanza & - & - & 36 & - \\
\hline Total & 245 & 397 & 202 & \\
\hline
\end{tabular}

Elaborado en base al Diagnóstico del Proceso de Actualización del Plan Maestro (2013-2018).

* Instituto Nacional de Estadística e Informática (INEI), 2017. Censos Nacionales de Población y Vivienda 2017.

** Según el Instituto del Bien Común \& Centro Peruano de Estudios Sociales, 2016. Directorio 2016 Comunidades Campesinas del Perú.

Se observa en la figura 2 que las comunidades de Mishana y Anguilla están ubicadas en las riberas del río Nanay. En cambio, para poder acceder a las demás se tiene que recorrer las quebradas afluentes al río Nanay, por lo que demanda más tiempo de traslado, consecuente a ello mayor utilización de combustible.

5 Decreto Supremo №006-99-AG expedido por el Ministerio de Agricultura. 


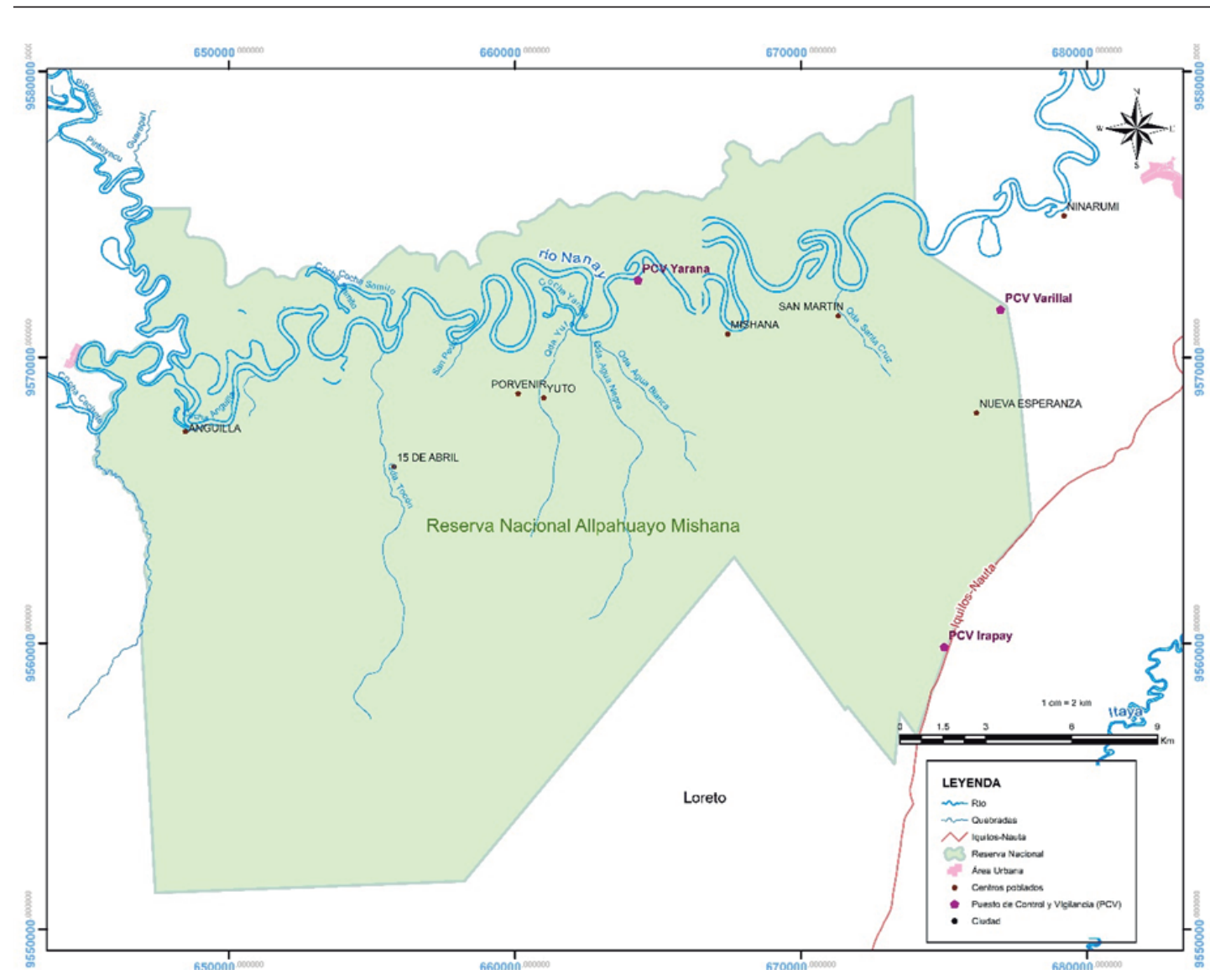

Figura 2 - Mapa de ubicación de los puestos de control y vigilancia y comunidades campesinas de la RNAM.

Fuente: Elaborado por Aguirre (2019)

Puesto de control y vigilancia Yarana, lunes 17 de enero de 2019.

Llegamos en la tarde del día anterior en yate; duró una hora y media de recorrido desde el centro poblado Ninarumi hasta llegar al puesto de control y vigilancia Yarana. El día 17 de enero por la mañana los guardaparques oficiales: Casiscley Pezo y German López nos brindaron algunos conocimientos sobre la flora y fauna y algunas indicaciones sobre el patrullaje con dirección a las comunidades ${ }^{6}$ de El Porvenir y San Juan de Yuto.

\section{Hacia la comunidad campesina El Porvenir, lunes 17 de enero de 2019.}

El patrullaje consiste en recorrer diferentes rutas en el área de la reserva, por donde vamos observando y fiscalizando si existe alguna infracción o actividad de extracción de recursos naturales, en este caso nos dirigimos hacia la comunidad El Porvenir y la comunidad de San Juan de Yuto.

Aquellos recorridos eran para nosotros (los guardaparques voluntarios) como un reconocimiento del área. Por lo cual, aún no se desarrolló las entrevistas a las autoridades, pero sí las observaciones y algunas preguntas libres a los pobladores.

Para poder llegar a estas comunidades, se navegó en bote o peque peque a través del río Nanay, partiendo desde el PCV de Yarana, hasta llegar a la quebrada Moena (ver figura 3). Al inicio de la quebrada, estaba ubicado un letrero donde decía "El Porvenir" (este recorrido duró 1 hora aproximadamente).

6 Según el Instituto del Bien Común \& Centro Peruano de Estudios Sociales (2016). Directorio 2016 Comunidades Campesinas del Perú. 


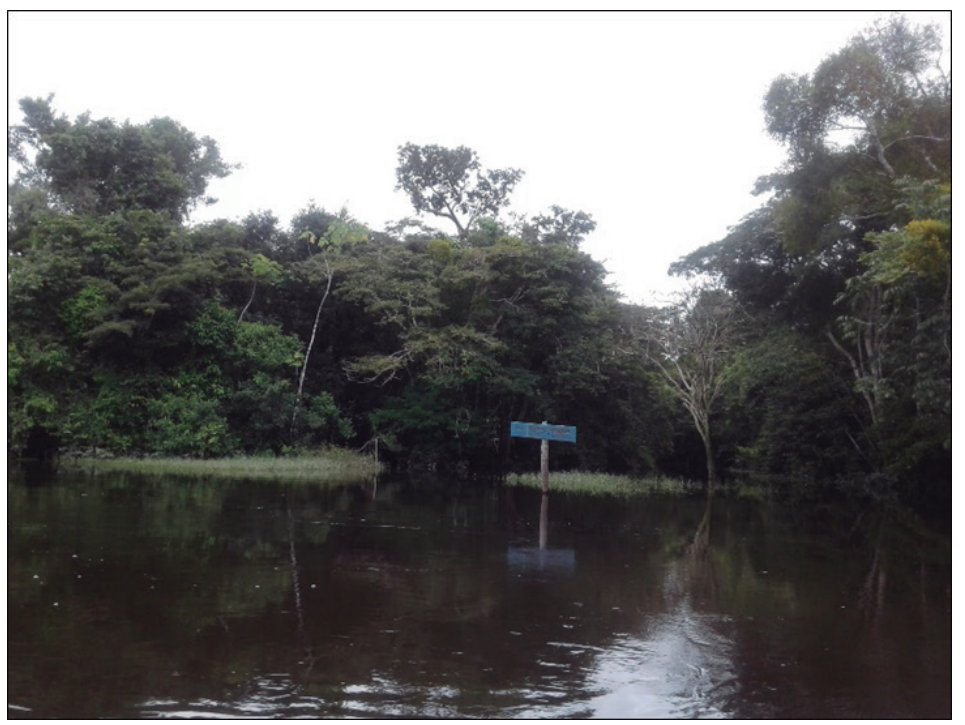

Figura 3 - Letrero del centro poblado El Porvenir. Fuente: Aguirre (2019)

Para llegar a la comunidad El Porvenir recorrimos la quebrada Moena. En este recorrido se evidenció por parte de los guardaparques, el conocimiento del área, de la flora y fauna de la reserva (ver figura 4):

En el transcurso del recorrido, que duró 20 min. aprox. el gp. German nos indicaba algunas especies de flora con sus respectivos usos, por ejemplo, el "aceite caspi" (para la construcción), Machinmangue, carhuasca y la pishirina. Llegando a un camino de trocha continuamos a pie dirigiéndonos a la comunidad, donde el recorrido duró en promedio 10 minutos.

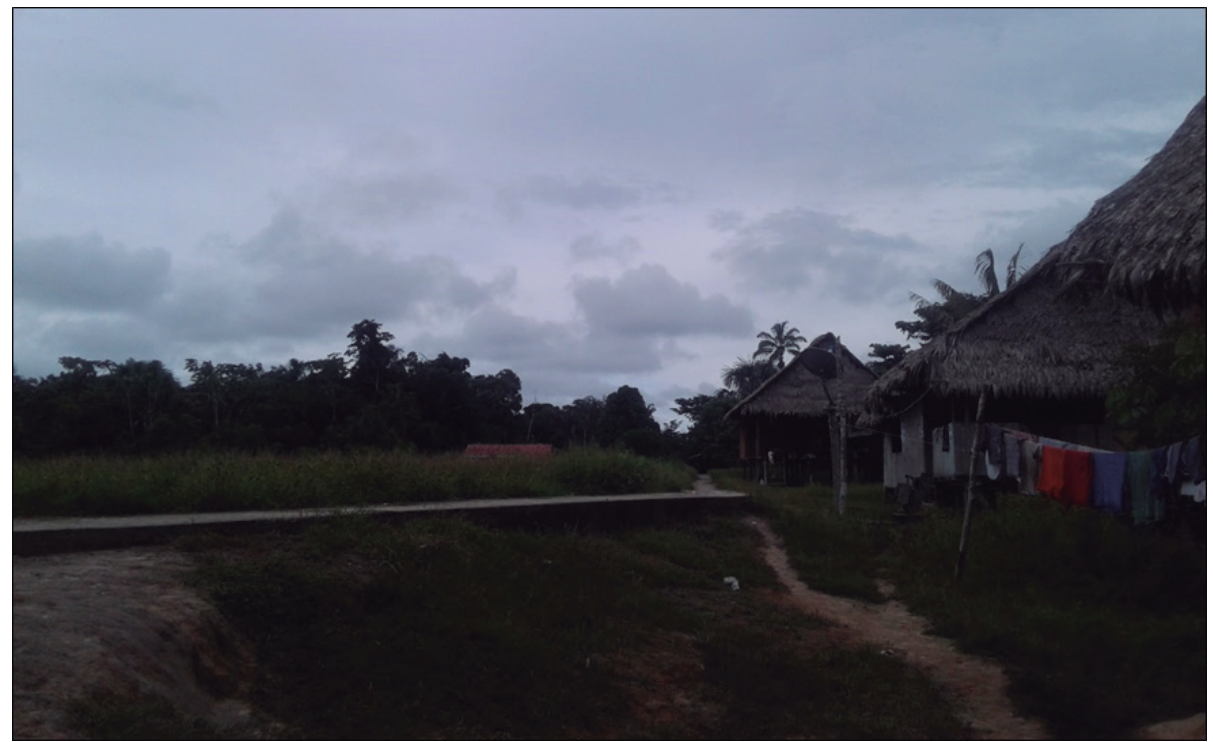

Figura 4 - Centro poblado de El Porvenir. Vista desde la llegada de la quebrada Moena. Fuente: Aguirre (2019)

Sin embargo, no solo el conocimiento era acerca de estos aspectos sino también el conocimiento de las diversas problemáticas que se desarrollaban en las comunidades.

Al llegar a la comunidad, después de recorrer 1 hora y 30 min. aprox., el gp. German nos informaba sobre la situación de esta comunidad. Nos indicó el lugar del centro de acopio, donde el pez de franja negra, que es ornamental, era criado por los pobladores. Como también nos informó que en el colegio "El Porvenir", el profesor solo asiste 10 días al mes 
en promedio, siendo este el único que viene a enseñar y direccionar el colegio. Por otra parte, también nos enseñaba algunos frutos de los árboles que estaban cercanos, donde pudimos reconocer al macambo, el aguaje, la tizana y el huasaí.

Los guardaparques preguntaban a los lugareños sobre cómo estaban la situación en la comunidad, mostrándose otras evidencias como el poder quedarnos los siete guardaparques en una casa o el de invitarnos alimentos de la zona, ello podría demostrar las buenas relaciones que mantienen los guardaparques con los pobladores.

\section{Hacia la comunidad campesina San Juan de Yuto, lunes 17 de enero de 2019.}

Para llegar la comunidad San Juan de Yuto, se caminó de 10 a 15 minutos. Este camino era trocha, donde también se tuvo que pasar por tres provisionales puentes en las tres quebradas que eran hechos con tronco de los árboles.

Una vez que llegamos a la comunidad de San Juan de Yuto, el gp. German nos presenta a un poblador residente de esta comunidad, al señor Alfonso Vásquez. Por lo tanto, nos presentamos como estudiantes y vertimos algunas preguntas libres; donde en las respuestas se evidencia una forma de reclamo de las condiciones precarias en la que se encuentran. De la cual, podemos rescatar las siguientes acepciones:

Indica el sr. Vásquez (2019) ${ }^{7}$ que los pobladores de Yuto se dedican a la crianza de alibinos o pez de franja negra (manera no tecnificada, en este caso eran criados en tina). También se dedican a la agricultura, a la "madera" (extracción para venta o uso propio) [...] Con respecto a los límites que tenían que respetar, consistía en una obtención como máximo de 300 maderas cada 3 meses con la condición de que provengan de árboles caídos naturalmente. Por lo que expresa el poblador, ante este límite, "como para poder sobrevivir", es decir, lo que pueden extraer es solo para su subsistencia [...] Entorno a la población, hace mención que la migración es constante, "antes eran cerca de 70 habitantes, pero por las dificultades, ahora son 35 personas". Y a su vez exclama: "el Estado está para servirle a él".

Sin embargo, en una cuestión personal del poblador, nos responde el motivo de su permanencia en la comunidad: "Estoy aqui porque me he acostumbrado a este ambiente, a comer las cosas frescas, a un clima suave y su tranquilidad". Esta última expresión que manifestó el poblador proviene de una buena la relación de su persona con el medio natural, más una relación de conflicto con la entidad estatal a consecuencia de las deficiencias y limitaciones de oportunidades percibidas en su comunidad.

\section{Hacia la comunidad campesina Mishana, sábado 26 de enero de 2019.}

Para llegar a la comunidad de Mishana, desde el PCV Yarana, se demora aproximadamente 10 minutos en el yate de la reserva.

$\mathrm{Al}$ darnos cuenta que los guardaparques conocen a la mayoría de los pobladores de las comunidades, el gp. oficial Jeder Pizango nos ayudó a comunicarnos con el teniente gobernador de la comunidad Mishana. En esta corta entrevista estaban presentes dos pobladores, tres guardaparques oficiales y los tres guardaparques voluntarios, mostrándose un espacio de intercambios de experiencias e información.

$\mathrm{Al}$ entrevistar al señor Fidencio ${ }^{8}$, teniente de la comunidad, nos responde con respecto a un posible desarrollo de su comunidad:

"Hay acuerdos que la comunidad misma desordena las cosas porque cree que uno está sacando ideas, cuando esta idea es mediante un acuerdo de todos, pero no lo cumplen por no estar presente cuando están estos acuerdos se realizan y por interés o motivos personales

7 Vásquez, A. (2019). Entrevista al poblador de San Juan de Yuto.

8 Fidencio, A. (2019). Entrevista al teniente municipal de Mishana. 
de algún poblador" [...] Es así que mencionó que un medio para avalar algún acuerdo es mediante un acta. Ello constata que si algún poblador no asiste a la reunión ya sea de la comunidad o los pobladores con la jefatura de la reserva, existe un documento firmado que todos deben cumplir [...] La relación que tiene la comunidad con las autoridades es por los desconciertos que hay de la jefatura hacia la comunidad, donde señala que, estas muchas veces no se hacen presente, aun comprometiéndose mediante un documento. También nos señaló la señora Alicia (pobladora de la comunidad), que se comprometen a una hora, y esta es incumplida, no asistiendo o viniendo 2 hasta 5 horas después.

Ante la entrevista se evidencian dos principales problemas. Primero los conflictos que existen en su misma comunidad por el deficiente interés de algunos pobladores ante los acuerdos que se establecen a nivel comunal. Y la segunda, con respecto al conflicto que mantiene con la jefatura, donde si logran reunirse, pero con una mínima cantidad de pobladores por la deficiente responsabilidad de la jefatura. Por lo tanto, los resultados de las reuniones no alcanzan a participar ni informar a la mayoría de los pobladores, de los cuales los tomadores de decisiones se reducen a un grupo mínimo generando especulaciones.

Al final el gp. le hizo llegar al teniente el documento donde se le invita a la reunión de trabajo para el miércoles 30 de enero (documento proveniente de la jefatura), ahí se estableció una agenda de trabajo. Por lo cual, el teniente firma para avalar el documento de invitación.

$\mathrm{Al}$ observar, la jefatura usa documentos donde sustenta las reuniones $\mathrm{u}$ otros tipos de acuerdos, pero al contrastar con la población, la mayoría indicó que no cumplen porque asisten a destiempo, asisten en otro día o no asisten.

Continuando con nuestras labores, antes de dirigirnos a la casa del agente municipal.

El gp. Jeder nos mostró el centro de acopio (tipo una casa prefabricada) de la crianza de pez banda negra o también llamado alibino (la misma actividad que las comunidades de Yuto y El Porvenir). Observamos que había varias peceras (un promedio de 18) y una manguera para el abastecimiento de agua, pero solo en una de ellas estaban con estas especies de peces.

El gp. Jeder como el gp. German muestra también el conocimiento no solo de la reserva sino también de las actividades que realizan las comunidades. De lo anterior, también se evidencia que ha existido o existe un proyecto no consolidado, ya sea por la responsabilidad de la jefatura, por la falta de dedicación de los pobladores o por ambos casos.

Nos dirigimos con el gp. Jeder a la casa del agente municipal para que le pueda entregar la invitación, por lo que logramos realizarle la entrevista.

Menciona Sergio Lozano (2019) :

"las problemáticas con reserva son para la obtención de los recursos", donde propone "que el estado fortalezca más a los guardaparques capacitándolos en el tema de infracción, en los recursos maderables, de pesca y en todos los recursos" [...] Con relación a la jefatura menciona: "nos ayuda con los puestos de vigilancia, ya que en esta zona hay bastantes infractores; las problemáticas que ellos tienen son problemáticas nuestras" [...] Ante la pregunta de ¿cómo ellos perciben los recursos que les ayuda manejar la reserva?, Sergio responde: "es como un refugio manejable, anteriormente se sacaba indiscriminadamente, sin control y lo depredaban" [...] Continuando con la pregunta con respecto a lo anterior, pregunto si todos los pobladores piensan de manera similar y si ha sido favorable la creación de la reserva, por lo que me responde que "la creación de la reserva es favorable, porque otras personas ya estaban depredando, pero no casi todos pueden asimilar al mismo tiempo que esto nos favorece, tienen diferentes grados, es duro convencer a la gente".

9 Lozano, S. (2019). Entrevista al agente municipal de Mishana. 
Con estas acepciones podemos inferir que existe una regular relación entre la población y la jefatura, ya que faltaría resolver algunas debilidades para una mejora tanto comunal como el área reservada. Pero también se evidencia un notorio conflicto entre los pobladores de la misma comunidad.

La preocupación de Sergio es también por el ámbito de obtener algún ingreso económico, por lo que menciona sobre la crianza de la banda negra, que "recién se ha empezado, pero esto es pasajero, igual que paso con la raya tigre, en cada año que pasa baja de precio, al principio costaba 500 soles y después de 4 años ya estaba 80 soles, esto es porque los que llevan, hacen que se reproduzcan en otro lugar". Al ser una oportunidad de venta de un nuevo producto corre riesgo de que no sea permanente y más aún si este no es patentado.

Prosiguiendo con la entrevista a Don Sergio, nos dio la curiosidad sobre la permanencia en la comunidad y no en la ciudad, por lo que nos responde: "la ciudad es bulliciosa, cuando voy a Iquitos me siento enfermo, con calor, aquí me siento tranquilo, despejado, libre, aquí es vida. Mishana tiene de todo, madera, frutos silvestres, peces, las plantas medicinales es abundante, esto es una farmacia".

Es así que, cabe la teoría que los pobladores de todas las comunidades permanecen en estas por los mismos motivos, ya que de similar forma nos mencionó el señor Alfonso de la comunidad de Yuto, aunque según la tabla 1 tiene como tendencia a la disminución poblacional.

\section{Hacia la comunidad campesina El Porvenir, martes 05 de febrero de 2019.}

Al regresar por segunda vez a la comunidad El Porvenir a cargo del gp. oficial Jeder, se observó una caseta abierta donde extraían agua. Este caño, a la cual se tenía que aplicar fuerza para la obtención del agua, era el único servicio para todos, es decir una fuente de abastecimiento de agua para toda la comunidad. Nos informaron los pobladores que este proyecto lo realizó una ONG y que este recurso cumplía con los estándares para su uso potable.

Otros de los servicios en la comunidad es la electricidad, la cual es generada por el almacenamiento de energía en las baterías que están conectadas a los paneles solares. Pero este uso de la electricidad se pudo observar en la noche en solo tres casas, ya que todos no poseían este servicio.

Gracias al gp. Jeder pudimos entrevistar a Beatriz López ${ }^{10}$, la teniente gobernadora de la comunidad de El Porvenir. Al ingresar a la sala de su casa fuimos bien recibidos, tomamos asiento y pudimos ser testigo de los reclamos que le hacía llegar al gp.

Para que puedan vender la madera extraída, indica la señora que "tenemos que esperar 15 a más días para la supervisión del producto. Uno se les comprende que no tienen bien el tiempo, pero nosotros también necesitamos todos los días ...si en un mes no se vende ningún producto, no se gana ni un sol, y ¿cómo se sobrevive?, es la realidad que se vive en todas las comunidades" [...] Al momento de preguntarle iqué recursos utilizan?, nos responde: "los recursos que utilizamos es madera, hojas de irapay, pesca, recién el año pasado se ha iniciado la actividad de banda negra... los varillales hace como 12 o 13 años eran aprovechados, quedaron en descanso, ahora ya se han recuperado bastante, pero la distancia es lo que nos limita a ir" [...] Prosiguiendo a preguntar sobre la creación de la reserva, Beatriz nos dice: "al principio de la creación de la reserva nos había chocado ... cuando no había reserva otra gente venía y extraían los recursos y ya no había para nosotros ... queramos o no queramos hemos aprendido a trabajar con manejo, para hoy mirar que a nosotros y a los animales nos favorece ... hemos caminado contigo Jeder, 5 horas y hemos visto tanta riqueza, los árboles, los animales" [...] Con respecto a la jefatura, la teniente mantiene una incomodidad al decir: "el jefe nuevo hasta ahora no viene a estar presente en una reunión con el pueblo y todos dicen que venga el jefe porque 
casi nadie lo conoce en las comunidades ... él tiene que venir a oír las inquietudes de los moderadores, de la realidad que se vive aqui ... y iquiénes son los que reciben todas las quejas?, son ustedes, los guardaparques... si nosotros empezamos a trabajar de mano a mano vamos a llevarnos bien".

Ante aquella respuesta, podemos interpretar que existen limitaciones de aprovechamiento de los recursos por las normativas de la reserva, donde están limitaciones dificultan una mayor actividad económica. Por otro lado, la comunidad mantuvo buenas relaciones con el guardaparque ya que pudo expresarle el problema de la relación que deberían guardar con la jefatura.

Al final el guardaparque le hizo llegar a la teniente el documento donde se le invita a la reunión de trabajo para el miércoles 30 de enero, donde se establece una agenda de trabajo, por lo cual la teniente firma para avalar el documento de invitación.

Como indicó la señora Alicia, que hay antecedentes que el actual jefe no se apersona, existe una disconformidad ya que, si vuelve a suceder, ellos como comunidad no podrían exponer sus dificultades, problemáticas, propuestas de solución o un acuerdo directo. Caso diferente que sucede en la comunidad de Mishana.

\section{Hacia la comunidad campesina San Juan de Yuto, martes 05 de febrero de 2019.}

Al dirigirnos a la comunidad campesina San Juan de Yuto, no pudimos encontrar al teniente gobernador, más dejamos el documento de invitación a un poblador.

Entonces, al preguntarle al poblador con relación al manejo de los recursos naturales, nos responde que este es "múltiple", nos daba como ejemplo de su persona, que él realiza la pesca, la agricultura, produce leña, hace construcción, entre otras actividades.

El conocimiento de los bosques varillales y su importancia de su conservación es percibido, ya que nos menciona el poblador que este ecosistema tiene "15 años de recuperación". De este bosque extraen el llamado "palo redondo" y para llegar a este bosque tienen que realizar una larga caminata.

Podemos pensar hasta aquí que, los pobladores tienen como sustento básico los bienes comunes (anteriormente llamados recursos naturales) que son su fuente de alimento, ingreso económico muy restringido por tratarse dentro de una reserva, donde además forma parte de su cultura. De los cuales, en la jefatura existen debilidades ante el trabajo en conjunto con la población. Pero al escuchar al siguiente representante, podemos interpretar otro tipo de relación muy contraria a la anterior.

Al acercarnos al agente municipal, Señes Piña ${ }^{11}$, que le encontrarnos al momento que nos estábamos yendo, nos ayudó con algunas interrogantes: Sobre los bosques varillales, nos indicó que "son bosques de arena blancas, lo conservamos, lo cuidamos para que no entren otras personas que quieren sacar madera o arena, estamos trabajando con la reserva, esta hacía reuniones o talleres ... la madera del bosque lo trabajamos poco, cuando lo extraen lo dejan descansar 5 años ... todo lo que nosotros trabajamos, damos todos los informes del trabajo de extracción sea de madera, hunguragui, aguaje, frutas" [...] Ante el beneficio o perjucio de la creación de la reserva al principio si les habia perjudicado por hacer "las documentaciones, todo el trámite de solicitar, pero ahora es bueno porque antes la gente de afuera se metía por todos lados ... por una parte, estamos más tranquilos, ya no vienen a sacar nuestros recursos" [...] Sobre alguna generación de ingresos nos mencionaba que comercializan los peces Alibinos, discos, escalares, que son peces ornamentales.

Ante esta corta encuesta, ninguno de los pobladores mostró su situación crítica en la que vivían, quizás por la falta de tiempo para poder realizar más preguntas. Aun así, recordando la primera visita al señor Alfonso de esta misma comunidad, se concluye una relación conflictiva ya que después dieron a conocer que la reunión que había 
establecido el jefe de la reserva (ver figura 5), no se cumplió. Por lo que podemos colegir que, el señor Peñes hacía referencia a las anteriores autoridades de la jefatura, tomando recién el cargo el actual jefe el 01 de agosto de $2018^{12}$.

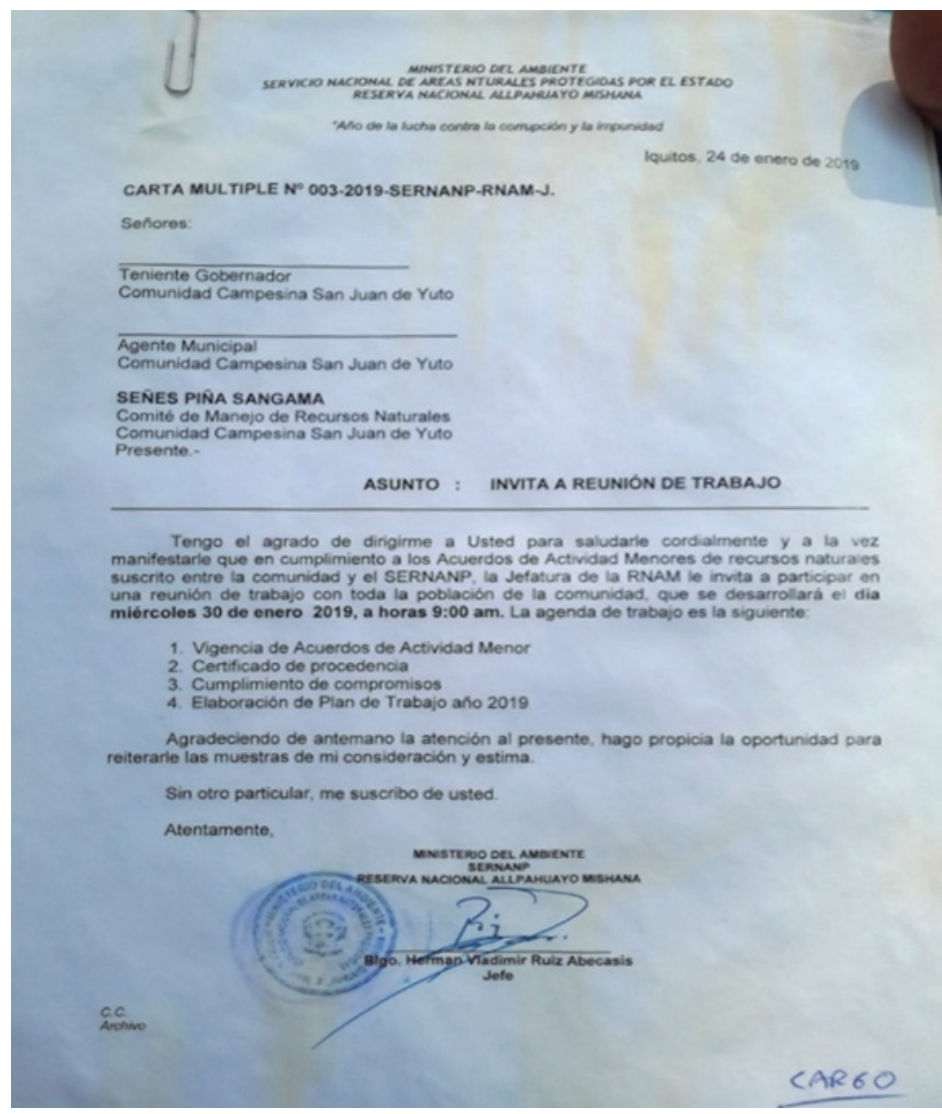

Figura 5 - Carta de invitación de la jefatura de la RNAM.

Fuente: Aguirre (2019)

\section{Observación de un problema de gestión, martes 19 de febrero de 2019.}

Se identificó un problema de gestión a nivel jefatural ante una visita de turistas extranjeros.

Al medio dia, tres turistas llegaron en un peque peque de un poblador al PCV Yarana. Aquellos turistas nos mencionaron que habían hablado con un guardaparque en la oficina del Sernanp. Lo que ellos entendieron es que no había ningún problema en quedarse en este puesto y que los encargados de este puesto les ayudarían a conocer el lugar y llevarlos al puerto de donde ellos habian venido. Pero al realizar la llamada del guardaparque German al jefe de la reserva, le manifestó que no tenía ninguna información de los turistas, aun así, dio la orden de que se podian quedar solo un día, más no realizar ningún recorrido con el yate de la reserva. Esta respuesta se les hizo llegar a los turistas de una manera amena, siendo estos impresionados porque el guardaparque de la oficina no les hizo llegar ningún conocimiento sobre ello. Al verse afectados económicamente, ya que se planteó como solución que la comunidad Mishana le haría recorrido como el regreso al puerto de Llanchama a un bajo costo, decidieron retirarse en el mismo instante, por lo que se les buscó la solución de solo conseguir un peque peque que los llevara al puerto de regreso. Este servicio fue brindado por un poblador de la comunidad de Mishana, ya que, también estaban descontentos, debido a que, en la oficina no se le brindó una buena información al detalle de los costos ni recorridos [...] Una pobladora de la comunidad mencionó que

12 Resolución de Presidencia № RP 185-2018-SERNANP. Recuperado de: https://www.gob.pe/institucion/sernanp/normas-legales/423033-rp-185-2018-sernanp 
ya se había acordado con la jefatura, que ellos (los de la comunidad) brindarían servicios turísticos, por lo que los turistas tenían que llegar primero a la comunidad y no al PCV Yarana, razón por la cual no se les fue indicado en el local [...] Los turistas mencionaron que eso no era lo que les informó el guardaparque de la oficina del Sernanp ${ }^{13}$.

Desde un punto de vista personal, este acontecimiento resultó perjudicial tanto para los turistas, para la comunidad de Mishana y para la RNAM. Es así que, se puede analizar que es deficiente la organización entre la comunidad de Mishana y la jefatura para el ámbito del turismo (en este caso), debido a que existe un Plan Maestro (20132018) ${ }^{14}$ desfasado y a que no existe aún un proyecto sobre este ámbito.

\section{Hacia la comunidad campesina de Mishana, miércoles 20 de febrero de 2019.}

Se realizó el patrullaje, donde se abarcó los terrenos de los pobladores de la comunidad de Mishana y otros pertenecientes a las personas no residentes del área. Este recorrido duró aproximadamente 6 horas. Partimos desde el núcleo urbano de la comunidad hacia la zona de bosque primario y secundario.

El trayecto consistía en recorrer el futuro circuito turístico (en ese momento se notaba un camino de tierra muy angosta resultado por las constantes pisadas de las personas bordeado de plantas y árboles) hasta la quebrada San Pedro [...] Los gp. Casiscley y German nos compartieron sus conocimientos de la flora y fauna, por lo que no detallaré estos aspectos, más si mencionaré sobre los temas sociales [...] Un problema que se presenció fue a pocos metros del circuito turístico. Se observó que un poblador cortaba los troncos de madera y que a su vez dos niños traían desde el bosque cargando otros troncos al mismo lugar. Este hecho según los guardaparques no debería ocurrir, ya que se había acordado que cerca del circuito turístico no debería realizarse actividades como estas (producción de leña). El segundo problema de este mismo hecho fue sobre la extracción del recurso maderable, proveniene de un bosque secundario, de los árboles de zancudo caspi y cunchi mohena.

Se deduce dos cosas ante esta situación: la primera es que los pobladores no están debidamente informados de los acuerdos de la jefatura con la comunidad, o que aún informados no cumplen con lo preestablecido.

Al regresar se observó que el guardaparque German preguntó a dos personas de la comunidad (en diferentes tiempos) de qué había pasado con el señor con el que estaba produciendo leña, explicándole a su vez que ya había un acuerdo de por medio (sobre la no realización de las actividades cerca al circuito y extracción de recursos en bosques secundarios), siendo este acuerdo de beneficio para toda la comunidad y preservación de los bosques. Estas dos personas respondieron quiénes son los familiares del señor.

Ello evidencia la preocupación y responsabilidad de los guardaparques para informar a la jefatura.

\section{Puesto de control y vigilancia Irapay}

El puesto de control y vigilancia de Irapay se encuentra en el kilómetro 28 de la carretera Iquitos-Nauta, a 40 minutos aprox. de la ciudad de Iquitos.

En este puesto de control se presenta un ecosistema totalmente distinto al de Irapay, ya que, es un ecosistema terrestre con quebradas ubicadas a varios kilómetros a diferencia del puesto de Irapay que es básicamente un ecosistema acuático debido al río Nanay y sus afluentes. Debido a ser un ecosistema terrestre los recorridos

13 Sernanp: Servicio Nacional de Áreas Naturales Protegidas por el Estado.

14 Resolución presidencial № 112-2012-SERNANP. Plan Maestro (2013-2018). Disponible en: http://old.sernanp.gob. pe/sernanp/archivos/biblioteca/planes_maestros_2014/Plan\%20Maestro\%202013\%20-\%202018\%20Allpahuayo\%20 Mishana\%20ver\%20aprob.pdf 
son a través de motos lineales, donde también existen limitantes debido al uso del combustible.

\section{Hacia la comunidad campesina Nueva Esperanza, sábado 9 de marzo de 2019.}

Se realizó el patrullaje en una moto lineal con destino a la comunidad de Nueva Esperanza a cargo del guardaparque oficial Neysser Pinedo. En medio de camino fuimos a una casa, la cuál era muy distante al núcleo urbano de la comunidad. En ella se encontraba solo el señor Esaut López ${ }^{15}$, por lo que se le preguntó sobre sus actividades y sobre la reserva.

Las actividades que practica son la agricultura y piscicultura, donde el poblador indica que no es apoyado por la reserva, es decir el solventa por sus propios medios para el desarrollo de sus actividades [...] Ante la pregunta de la creación de la reserva, menciona que "la creación de la reserva es buena porque la gente de afuera venían a depredar los bosques, desde su creación ya no depredan los bosques".

Se evidencio dificultad en continuar expresando sus respuestas, pero podemos inferir que las comunidades continúan trabajando independientemente de la jefatura en relación a la extracción de los bienes comunes, sin embargo, estos pobladores tienen limitantes donde a veces conlleva a una fiscalización que no pueden abastecerse la propia jefatura para terminar a tiempo todas sus funciones.

Luego de la conversación breve con el poblador, nos dirigimos al núcleo urbano, pero no se presenciaban personas, por lo que nos dirigimos a una casa donde el gp. conocía al dueño, al llegar encontramos una señorita que nos indicó que el dueño no estaba, por lo que tampoco nos pudo brindar el apoyo de la encuesta.

Continuando el camino del patrullaje al albergue "AYAMADRE". Este una zona alejada del núcleo urbano de la comunidad, donde se realizan algunos rituales, (por ejemplo, un ritual con la bebida del Ayahuasca). Fui testigo de la conversación sobre los informes de los terrenos entre el guardaparque y la dueña del Albergue. Esta conversación indicaba que existe un problema de venta de terrenos, aun siendo propiedad de la reserva. Para inspeccionar este hecho, nos dirigimos unos 2 kilómetros, donde se observó una estructura de otro albergue que recién estaban construyendo, mas no hubo ninguna persona en el área (ver figura 6). Al ver este hecho se presenció la preocupación del gp. por lo cual registramos la ubicación con el GPS y tomamos algunas fotografías para después informar al jefe de la reserva.
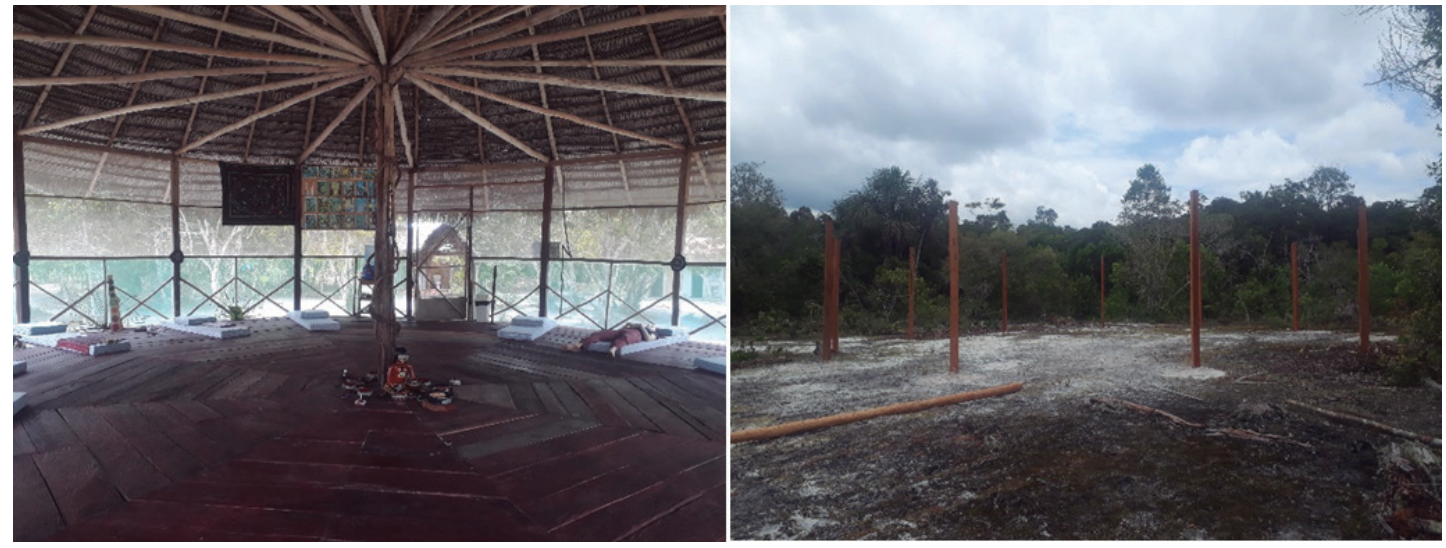

Figura 6 - Albergues. El de la izquierda es el albergue autorizado llamado "Ayamadre", el de la derecha es el albergue en construcción sin autorización

Fuente: Aguirre (2019)

15 López, E. (2019). Entrevista al poblador de Nueva Esperanza. 
La existencia de problemas de propiedad de terrenos son otras cuestiones por resolver, cuya competencia es directamente con el Sernanp y los supuestos dueños que no son lugareños. Es así que se evidencia un conflicto que tendría que asumir la jefatura y los pobladores externos.

\section{Oficina de la jefatura de la Sernanp, lunes 11 de marzo de 2019.}

La oficina de la jefatura está ubicada en la Calle Tacna $N^{\circ} 432$, Iquitos - Maynas. Oficina de la cual lo conforman algunos jefes de las reservas de Loreto con sus personales respectivos como biólogos, ingenieros, voluntarios, entre otros.

Se realizó cortas entrevistas con los trabajadores: Hermán Ruiz ${ }^{16}$ (jefe de la reserva) y a Roger Alvarado ${ }^{17}$ (guardaparque oficial) como también observaciones de campo a los especialistas (biólogos e ingenieros), este último sin influenciar o generar cambios ante los hechos.

El jefe de la reserva nos menciona que existen concursos de proyectos para las reservas, por lo que este brindará un financiamiento para que se pueda desarrollar el turismo. Así también existen otras oportunidades por lo que se tiene que se tiene que realizar permanente seguimiento a los procesos [...] No se le realizó más preguntas porque se percibió una indisposición ya que contestaba con otros temas [...] Por parte del gp. mencionaba que su persona cumple más funciones en la oficina que en el campo debido a que el jefe le asigna tareas, como la representación de eventos, el manejo de documentos y redes de las comunicaciones. Por las observaciones sobre el gp. Roger, él está más relacionado con la jefatura de la reserva, ya que fui testigo al estar el PVC de Irapay, que el gp. venía con voluntarios de la universidad y con niños de instituciones educativas para realizar el recorrido del circuito turístico (que duraba en promedio 30 minutos) aplicando una temática de educación ambiental [...] Por otro lado, también se observó que los especialistas profesionales (biólogos, ingenieros u otros) mantienen funciones permanentemente en la oficina. La experiencia que también pude observar fue cuando mi persona estuvo en el PCV de Yarana, los especialistas y el jefe fueron una hora a este puesto para después apersonarse a la comunidad de Mishana, mas no a las demás comunidades. Ello se constata (en otro patrullaje), ya que los pobladores de esta comunidad criticaron la falta de respeto al venir horas después de lo acordado. Mientras que las demás comunidades nos indicaron que los especialistas ni el jefe vinieron, y que a su vez habian sido perjudicados ya que los pobladores habían dejado de realizar otras actividades para la reunión que ya estaba acordada.

Acorde con esta experiencia con la jefatura y los que están directamente relacionados con ella, infiero que mantienen una relación de conflicto con las comunidades, ya sea por la deficiente responsabilidad con las mismas o por la concentración netamente de los proyectos para la reserva.

\section{Metodología para la elaboración del mapeo de actores según Tapella, E. (2007)}

La metodología propuesta por Tapella es basada en el enfoque de Pozo-Solis (2007) y EC-FAO (2006). Este enfoque está estructurado en 6 pasos, los cuales se sintetizan y caracterizan en la siguiente tabla (ver tabla 2):

Esta tabla de cinco columnas sintetiza, en la primera a los actores que fueron registrados en el trabajo de campo; en la segunda, los nombres de los actores; en la tercera, el rol que cumple cada actor en el área de estudio; en la cuarta, si los actores registrados estaban de acuerdo que le realicen encuestas o algunas preguntas para la recolección de datos; y en la quinta el nivel de poder que poseen los actores en la RNAM.

16 Ruiz, H. (2019). Entrevista al jefe de la RNAM.

17 Alvarado, R. (2019). Entrevista al guardaparque oficial. 
Tabla 2. Matriz de Actores de la RNAM

\begin{tabular}{|c|c|c|c|c|}
\hline $\begin{array}{l}\text { GRUPO DE ACTORES } \\
\text { SOCIALES }\end{array}$ & ACTOR & ROL EN EL PROYECTO & $\begin{array}{l}\text { RELACIONES } \\
\text { PREDOMINANTES }\end{array}$ & $\begin{array}{l}\text { NIVELES } \\
\text { DE PODER }\end{array}$ \\
\hline Profesional & $\begin{array}{l}\text { 1. Jefe de la } \\
\text { reserva: Herman } \\
\text { Ruíz Abecasis }\end{array}$ & $\begin{array}{l}\text { Es el profesional que se encarga de la } \\
\text { jefatura de la RNAM. }\end{array}$ & Indiferente & Alto \\
\hline Profesional & 2. Gp. oficial: Roger & $\begin{array}{c}\text { Es el profesional que se encarga de las redes } \\
\text { de comunicaciones }\end{array}$ & sin dato & Medio \\
\hline Gp. oficial & 3. Germán & Es guardaparque oficial de la RNAM. & A favor & Medio \\
\hline Gp. oficial & 4. Casiscley & Es guardaparque oficial de la RNAM. & A favor & Medio \\
\hline Gp. oficial & 5. Jeder & Es guardaparque oficial de la RNAM. & A favor & Medio \\
\hline Gp. oficial & 6. Neisser & Es guardaparque oficial de la RNAM. & A favor & Medio \\
\hline $\begin{array}{l}\text { Representante de El } \\
\quad \text { Porvenir }\end{array}$ & $\begin{array}{l}\text { 7. Teniente gob.: } \\
\text { Beatriz López }\end{array}$ & $\begin{array}{l}\text { Es el representante del centro poblado de El } \\
\text { Porvenir }\end{array}$ & A favor & Bajo \\
\hline $\begin{array}{l}\text { Representante de } \\
\text { Mishana }\end{array}$ & $\begin{array}{l}\text { 8. Teniente gob. : } \\
\text { Fidencio Sutac }\end{array}$ & $\begin{array}{l}\text { Es el representante del centro poblado de } \\
\text { Mishana }\end{array}$ & A favor & Bajo \\
\hline $\begin{array}{l}\text { Representante de } \\
\text { Mishana }\end{array}$ & $\begin{array}{l}\text { 9. Agente mun. : } \\
\text { Sergio Lozano }\end{array}$ & $\begin{array}{c}\text { Es el representante que se encarga de velar } \\
\text { por las necesidades de la comunidad para } \\
\text { comunicarse con el alcalde distrital de San } \\
\text { Juan Bautista }\end{array}$ & A favor & Bajo \\
\hline $\begin{array}{l}\text { Representante de } \\
\text { Yuto }\end{array}$ & $\begin{array}{l}\text { 10. Agente mun. : } \\
\text { Señes Peña }\end{array}$ & $\begin{array}{c}\text { Es el representante que se encarga de velar } \\
\text { por las necesidades de la comunidad para } \\
\text { comunicarse con el alcalde distrital de San } \\
\text { Juan Bautista }\end{array}$ & A favor & Bajo \\
\hline $\begin{array}{l}\text { Poblador de Nueva } \\
\text { Esperanza }\end{array}$ & 11. Esaut López & $\begin{array}{l}\text { Poblador dedicado a la agricultura y } \\
\text { piscicultura }\end{array}$ & A favor & Bajo \\
\hline $\begin{array}{l}\text { Poblador de San } \\
\text { Juan de Yuto }\end{array}$ & $\begin{array}{l}\text { 12. Alfonso } \\
\text { Vásquez }\end{array}$ & $\begin{array}{c}\text { Poblador dedicado a la agricultura y } \\
\text { piscicultura }\end{array}$ & A favor & Bajo \\
\hline
\end{tabular}

Fuente: Elaborado por Aguirre (2019)

A continuación, el gráfico de la relación de los niveles de poder e interés en el objetivo (ver figura 7).

Se describe del gráfico que existe un solo actor con el nivel de poder alto, por lo que mantiene las relaciones de fuerte colaboración solo con los guardaparques, más existe algunas relaciones puntuales con los pobladores de la comunidad, como también relaciones de conflicto con dos representantes y un poblador. El vínculo que poseen los guardaparques con las personas de la comunidad son relaciones buenas, como se podrá observar los guardaparques no mantienen ninguna relación de conflicto con los demás actores. Por otro lado, entre los guardaparques existe relaciones buenas excepto con el gp. 2, de la cual no se pudo determinar el tipo de relación. Este último no mantiene relación con lo demás con excepción del jefe de la reserva.

Se puede concluir que estas perspectivas de los tres actores: los pobladores de las comunidades campesinas, los guardaparques oficiales y la jefatura de la RNAM, manifiestan posiciones divergentes (buenas relaciones, relaciones puntuales y relaciones de conflicto). Como es el caso de las relaciones de conflicto entre la jefatura y los pobladores. Los pobladores se enfocan principalmente en los deficientes compromisos que manifiesta la jefatura ante las comunidades; los guardaparques expresan la preocupación del estado de las comunidades campesinas y el manejo de recursos naturales tanto de los pobladores y personas externas de la reserva; por último, la jefatura de la reserva se enfoca en los temas de gestión básicamente desde el local de la Sernanp para la obtención de futuras oportunidades para la RNAM. 


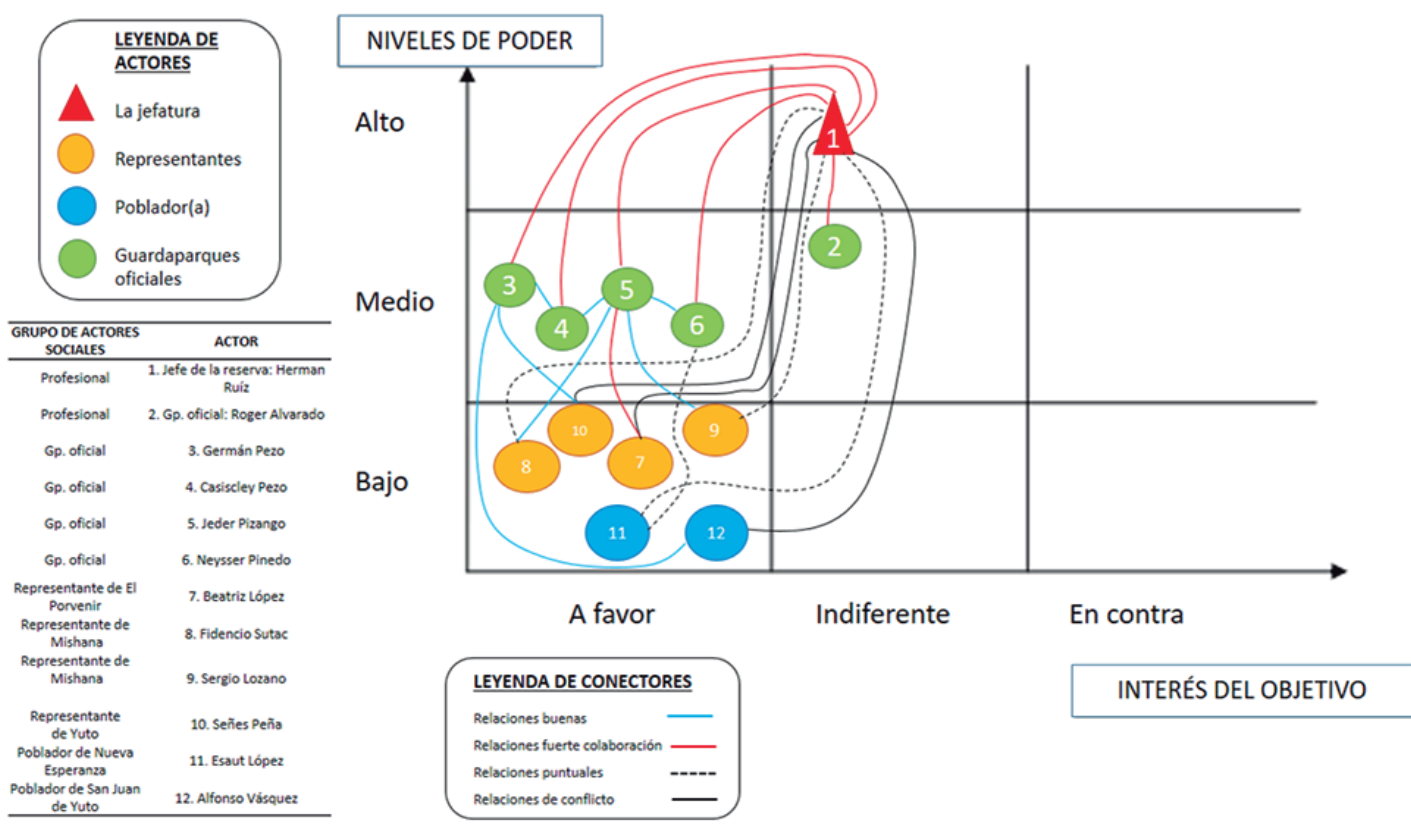

Figura 7 - Mapa de poder de la RNAM

Fuente: Elaborado por Aguirre (2019)

\section{Reflexiones finales}

Se analizó las relaciones que existen entre los tres actores; sin embargo, se debe incluir para un estudio a mayor detalle tanto las relaciones que tienen entre los representantes de las comunidades como las relaciones que se desarrollan entre los representantes y pobladores.

Es importante señalar que quien tiene el poder en el área de estudio, no involucra que toda gestión dentro de la reserva lo abarque la jefatura, como es en el caso de la educación con pésima calidad de las instituciones educativas ubicadas dentro de la reserva. No obstante, esta sí puede influenciar en desarrollar una temática de educación ambiental. Este ámbito radica en la toma de decisiones, por lo que es imprescindible conocer como el jefe mantiene sus relaciones con los otros actores.

Según el mapa de actores, los guardaparques oficiales son los actores estratégicos en el área de estudio, ya que estos no guardan ninguna relación de conflicto ni con la jefatura ni con los pobladores, aun ni entre ellos mismos. Cabría pensar de ¿cuáles serían las ventajas si los guardaparques son los actores estratégicos para un desarrollo de un proyecto?

\section{Bibliografía}

Anónimo (2018). Definition of point of view (2019). Disponible en: https://www.charlesclarke. org.uk/pdf-book/point-of-view-literature-definition[Fecha de consulta: 14/03/2019].

Hautamaki, A. (2015). Change, Event, and Temporal Points of view. ResearchGate. University of Helsinki, Helsinki, Finland.

Instituto del Bien Común \& Centro Peruano de Estudios Sociales (2016). Directorio 2016 Comunidades Campesinas del Perú.

Instituto Nacional de Estadística e Informática (2017). Censos Nacionales de Población y Vivienda 2017.

Real Academia Española (2001). Diccionario de la lengua española (2001). Disponible en: http://lema.rae.es/drae2001/srv/search?id=PBcqiKUvlDXX2s6mofda [Fecha de consulta: 14/03/2019]. 
Reserva Nacional Allpahuayo Mishana (2013). Diagnóstico del Proceso de Actualización del Plan Maestro (2013-2018). Iquitos - Perú.

Tapella, E. (2007). El mapeo de Actores Claves, documento de trabajo del proyecto Efectos de la biodiversidad funcional sobre procesos ecosistémicos, servicios ecosistémicos y sustentabilidad en las Américas: un abordaje interdisciplinario", Universidad Nacional de Córdoba, Inter-American Institute for Global Change Research (IAI). 